\title{
An Architectural Framework for Distributed Naval Ship Systems
}

\author{
Dorian Brefort, Colin Shields, Michael Sypniewski, Conner Goodrum, David Singer, \\ University of Michigan, USA \\ Agnieta Habben Jansen, Etienne Duchateau, Koen Droste, Ted Jaspers, Hans Hopman, \\ Austin A. Kana, Delft University of Technology, NL \\ Rachel Pawling, David Andrews, University College of London, UK \\ Mustafa Yasin Kara, Mark A. Parsons, Alan Brown Virginia Tech, USA
}

\begin{abstract}
This paper introduces a framework for analyzing distributed ship systems. The increase in interconnected and interdependent systems aboard modern naval vessels has significantly increased their complexity, making them more vulnerable to cascading failures and emergent behavior that arise only once the system is complete and in operation. There is a need for a systematic approach to describe and analyze distributed systems at the conceptual stage for naval vessels. Understanding the relationships between various aspects of these distributed systems is crucial for uninterrupted naval operations and vessel survivability. The framework introduced in this paper decomposes information about an individual system into three views: the physical, logical, and operational architectural representations. These representations describe the spatial and functional relationships of the system, together with their temporal behavior characteristics. This paper defines how these primary architectural representations are used to describe a system, the interrelations between the architectural blocks, and how those blocks fit together. A list of defined terms is presented and a preliminary set of requirements for specific design tools to model these architectures is discussed. A practical application is introduced to illustrate how the framework can be used to describe the delivery of power to a high energy weapon.
\end{abstract}

Keywords: Early-Stage Design; Distributed Systems; Complex Systems; Design Taxonomy 


\section{Introduction}

The increasing importance and complexity of interdependent distributed systems, people, and components incorporated into naval ships makes it necessary to describe them as architectures of complex systems. In this paper, the authors present and demonstrate a framework to describe the architectures of distributed naval ship systems that enables engineers to better address system design in early stage naval ship design. The framework is intended to provide a conceptual method of capturing the key attributes of a distributed ship system. Thus, the objective is to describe such a system, ensuring all important aspects are covered, as opposed to presenting a design process for the system.

A distributed ship system representation needs to be multifaceted and it is the interrelationships between the different representations, or architectures, that allows a full understanding of the system. The presented framework is applicable to one given system, and is designed to cover the aspects that are important when analyzing and describing that system. Three primary facets of a system are considered, the physical, logical and operational architectures. The architecture of a system is defined as the manner in which its components are organized and integrated. The physical architecture represents the spatial and physical characteristics of the system and of its environment. The logical architecture describes functional characteristics of the system, and the linkages between each component of the system. The operational architecture describes temporal behavior of a system, including human-system interactions to some extent. The architectural framework presented in this article thus provides a basis for describing and understanding the impact of the architectural properties of systems aboard a vessel on the vessel's performance. 
The effort to develop this framework was motivated by a need to better understand the impact of distributed systems on ship design, especially with regard to survivability.

The past half century has brought with it a radical change in the design and control of high-risk systems. Technological change has brought with it highly complex, automated, capable, but opaque systems (Reason, 1990). This growth in system complexity and interdependence has made systems significantly more difficult to understand and design, in part due to increased potential for emergent properties that only arise once the system is complete and in operation. This increased the opportunity for latent errors (i.e. design errors which can remain dormant for a long time before the right combination of factors align to make the error emerge) and potentially catastrophic consequences on the ship's operability (Britton, 2016; Slabodkin, 1998). The opacity of the systems' interrelations has led to an increased opportunity for cascading failures, compromising the survivability of the vessel. As noted above, the framework aims to better understand the multidimensional relations between distributed systems, which can decrease the design's opacity.

With the increasing number, complication, and resulting complexity of systems aboard vessels, the authors argue this framework will bring a major step forward in early stage naval ship design and analysis, addressing a significant gap in our ability to investigate the complex nature of future naval distributed system design at this crucial decision point. Section 2 outlines the problem and justifies the need for an architectural framework in the design of distributed systems aboard naval vessels, while Section 3 presents the framework itself, with its three primary architectures and their interrelations. Section 4 discusses requirements for analysis tools and 
recommendations for the architectural framework implementation. In Section 5, an example application is given involving the powering of a vessel's high energy weapon. The Appendix defines the key nomenclature used in this framework.

\section{Background: The Need for an Architectural Framework}

In the past several decades, technological developments have brought major changes to the way ships are designed and operated. Automation has pushed the boundaries of performance and increased the use of complex distributed systems aboard vessels. Although costly to introduce, automation has had an attractive payback to the maritime industry. The increase in complex systems and automatic monitoring systems has led to increased operational efficiency, increased crew morale and safety, and reduced maintenance cost (Ehlers et al., 2014). The advantages of all-electric powering over mechanically powered ships are also well documented, and have led many organizations to move towards all-electric ships. Some major advantages of all-electric powering were identified by Doerry (2014), including: increased power flexibility (the ability to shift power between ship systems as needed), increased power efficiency, and increased arrangements flexibility since prime movers are no longer restricted to the central position of the aft bottom decks.

The introduction of complex distributed systems and electric propulsion technologies aboard naval vessels has nevertheless significantly increased their complexity (Rigterink, 2014) and has left a gap in ship design methods and tools. The increasingly complex organizational and physical architectures of naval systems, with high interdependence between distribution systems, humans, and onboard components, are changing the design drivers and the focus of the naval architect. System integration is now as important as the traditional naval architecture disciplines 
since the arrangement of systems and usage of system interdependencies play a significant role in vessel cost (Dobson, 2014; Miroyannis, 2006), capability, and survivability (Doerry, 2006, 2007; Trapp, 2015). Thus, understanding the structure of the dependencies between various aspects of a distributed system and how they are best accommodated in the ship's physical architecture in the early stages of design is critical to the maturation of state-of-the-art vessel design (Brown and Waltham-Sajdak, 2015; Chalfant, 2015; Ouroua et al., 2007).

The changing design considerations of naval vessels that arise from the increased use of distributed systems have left designers with an inadequate set of tools for concept exploration (Doerry and Fireman, 2006; Kassel et al., 2010). Analyzing the implications of early stage design decisions on the physical attributes of the vessel only covers a limited aspect of early stage design - how components and discrete sub-systems within compartments are geometrically related, and how the resulting configuration affects the functionality and performance of the vessel. With simpler, less demanding vessels, designers were able to use their implicit knowledge to determine performance and interaction issues that could occur between systems in a given general arrangement. However, with the increased impact of distributed systems and smaller margins driven by the desire to further optimize designs, new methods are needed to help designers integrate vessel solutions. These need to reflect the interdependent functionality of components within a vessel, how the functionalities provided by the whole vessel and the component sub-systems will be achieved, and geometric relationships caused by an arrangement. To the authors' knowledge, no cohesive framework exists for evaluating how the coupling of these interrelated design aspects culminates in determining the overall system performance. 
Common methodologies for concept design of interacting ship architectures range from low fidelity parameterization based modeling to high fidelity simulation of systems (Andrews, 2012; Brown and Waltham-Sajdak, 2015; Chalfant, 2015; Chandrasegaran et al., 2013). Parametric methods perform well for evolutionary designs; however, their basis in previous data makes them ill-suited for the design of revolutionary vessels (Chalfant, 2015). Low fidelity simulation is also used for concept exploration in distributed system design. Trapp (2015) uses optimization on a multi-commodity flow network to explore a large state space and find a "minimum" cost of a vessel's integrated engineering plant with a given survivability constraint. His algorithm seeks the minimum cost survivability and avoids predicating a single solution. Cramer et al. (2009) use a genetic algorithm to solve minimax problems and applies it to the design of an integrated engineering plant with respect to survivability.

After the set of possible solutions is narrowed using low fidelity simulation, high-fidelity analysis of specific systems can be targeted. For instance, high-fidelity analyses have been performed to analyze the tradeoff between AC and DC electrical distribution systems (Chalfant et al., 2010), understand the impact of electrical weapons on power supply stability (Whitelegg et al., 2015), but also to understand the relationship between propulsion and maneuvering systems (Altosole et al., 2010). However, high-fidelity models often require a significant jump in design detail and can take up valuable time to model in early stage design, especially at a point in time where the chosen design solution has yet to emerge. The time issue can be addressed using tools that can easily produce and analyze distributed systems based on templates and product catalogues. The Electric Ship Research and Development Consortium (ESRDC) has developed the Smart Ship Systems Design (S3D) tool to perform high fidelity design and analysis of 
distributed naval systems (Chalfant, 2015). Fiedel et al. (2011) developed a cooling system design tool to analyze thermal loading and design appropriate cooling systems. This is a task which will become harder and more critical as the number of electrical systems aboard ships increases. The jump in design detail required for high fidelity models and templates still remains an issue, as they both strongly rely on previous solutions and assumptions, and on modeling detail which is based on decisions that can predicate the design, influencing it at a point in time where the chosen design solution has yet to emerge. This makes them ill-suited for concept design or for the creation of revolutionary designs like naval all-electric ships and radical ship configurations (Greig et al., 2009), where one should not fix large portions of the design while still conducting requirements elucidation (Andrews, 2011, 2013).

Addressing the architecture of naval distributed systems in novel vessel design is becoming a major component of concept exploration and is beset by technology uncertainty and concurrent mission development. Developing vessel concepts from legacy designs, tools, and fixed solution options significantly limits the designer's ability to take advantage of emergent opportunities and properly cope with evolving design requirements in early-stage design, when decision freedom is highest (Andrews, 2013). New methods are needed to guide the development of architectures of distributed systems from conception through early stage design and into more detailed effort without requiring leaps to high fidelity tools, while the ship design is far from fixed.

\section{Architectural framework for naval distributed systems}

The architectural framework of a system is defined as the manner in which its components are organized and integrated. This is the focus of this paper: to describe an architectural framework 
for naval distributed systems which accounts for the relationships concerning a specific distributed system and its relation within the ship's general arrangements, in the context of naval survivability. Specifically, the authors use the definition of The Open Group Architecture Framework (TOGAF), which defines and describes procedures for developing an architectural framework as:

"[An architectural framework is] a foundational structure, or set of structures, which can be used for developing a broad range of different architectures. It should describe a method for designing a target state of the enterprise in terms of a set of building blocks, and for showing how the building blocks fit together. It should contain a set of tools and provide a common vocabulary. It should also include a list of recommended standards and compliant products that can be used to implement the building blocks.” (TOGAF, 2011).

In general, a framework consists of the structure of a system, its properties and behaviors, and the relationships that exist within it. Architectural frameworks are often used to analyze and to describe the organization of systems like buildings, computers, or even human biology. They are well suited for any system with a large number of connected, interdependent components. For many applications, a well modeled architectural framework enables reuse of components to ease development and management for current and future programs or projects. In this sense, the following discussion aims to: 1) describe the architectural blocks used in this framework, 2) describe how these architectural blocks fit together, 3) provide a brief discussion of requirements for relevant analysis tools, and 4) establish the common vocabulary. 


\subsection{Primary architectures}

In the context of naval ships, the authors define the architectural framework as a conceptual model that describes the physical architecture, the logical architecture, and the operational architecture of vessels, together with the interrelationships between the three architectures. The physical architecture describes the spatial arrangement, the logical architecture describes information on the functional characteristics of the system, and the operational architecture contains information on the temporal behavioral characteristics of the vessel, in a given mission scenario. These primary architectures are discussed in more detail below. In section 3.2 , we describe how these architectural blocks fit together.

The physical architecture is a spatial architecture and it has two important classes of information: (1) the constraining architecture defined by the ship configuration and the relationships between spaces (essentially what spaces actually exist on the ship, and how they physically relate to one another), and (2) the physical attributes of components of a given distributed system and their locations relative to each other in the vessel. The physical architecture defines the organization of the overall layout of major spaces and thus the possible spatial configurations a given distributed system can take within these spaces. It creates bounds on the possible layout configuration of distributed systems. Example physical architectures are given in Figure 1. The radial configuration shown on the left exhibits a smaller footprint but has less potential for system routing redundancy than the ring configuration on the right. 

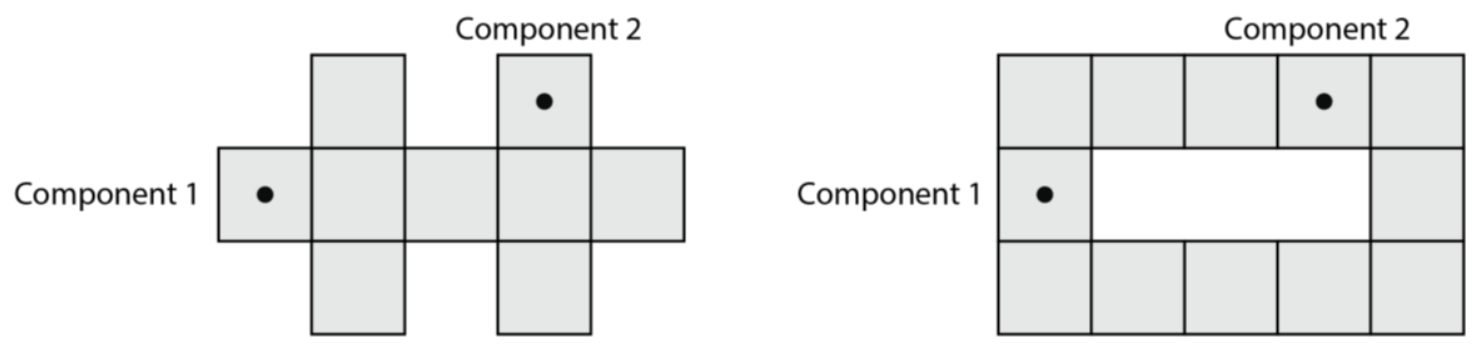

Figure 1: Physical architectures of distributed systems with varying characteristics.

The choice of style, as discussed by Andrews (2012) and Pawling et al. (2013, 2014), is a major driver of any ship design option and hence has a significant influence on the physical architectures. Style could be the basis of producing a set of design options, for the ship and a given distributed system, within it. Style refers to a set of options resulting from choices in design (e.g. the choice of a survivability zoning configuration) that are usually cross-cutting between various disciplines. Thus, two vessels with the same requirements can have very different characteristics and performances. For example, a common set of performance requirements for a frigate was once provided to both the UK and the US navies. Though the performance requirements were similar, the resultant UK and US designs were very different (Ferreiro and Stonehouse, 1991). These designs differed in size, displacement, number of decks, shape of the superstructure, etc. In other words: the physical architecture of the designs was different, though the high level operational requirements were identical (Pawling et al., 2014).

The logical architecture describes functional characteristics of the system, and the linkages between each component of the system. Logical architecture is a multidisciplinary term, and in this paper its definition is similar to the one used in information technology. Specifically, Desfray (2008) states that logical architecture, "addresses the information system seen 
macroscopically, by focusing on its main components, their interactions and the flows exchanged, and by structuring them by group into larger-scale modules." It is this essence of the term that the authors aim to apply to naval systems and arrangements. In that case, 'components' could refer to a machine system component and an auxiliary power source, while 'flows exchange' could comprise power distribution between power sources and sinks. Another example of a logical architecture may be a single line diagram describing the way components are connected or should be connected to each other to provide a given service or function. In the case of a ship's machinery system, this may detail that a system component must be connected to a main auxiliary power source and to multiple emergency power sources. Figure 2 shows an example logical architecture of distributed systems, represented as a multiplex network. The components are the same in both networks. The left one shows the relations in a cooling system and the right one shows the electrical power system. In the electrical power system, the radar is connected to the power source node and the backup power node. Thus, the radar has power redundancy. However, the chiller is not connected to the backup power source node. If the main power source fails, the radar will eventually become inoperable from overheating, and this relationship between power and cooling has not been captured by the individual systems.
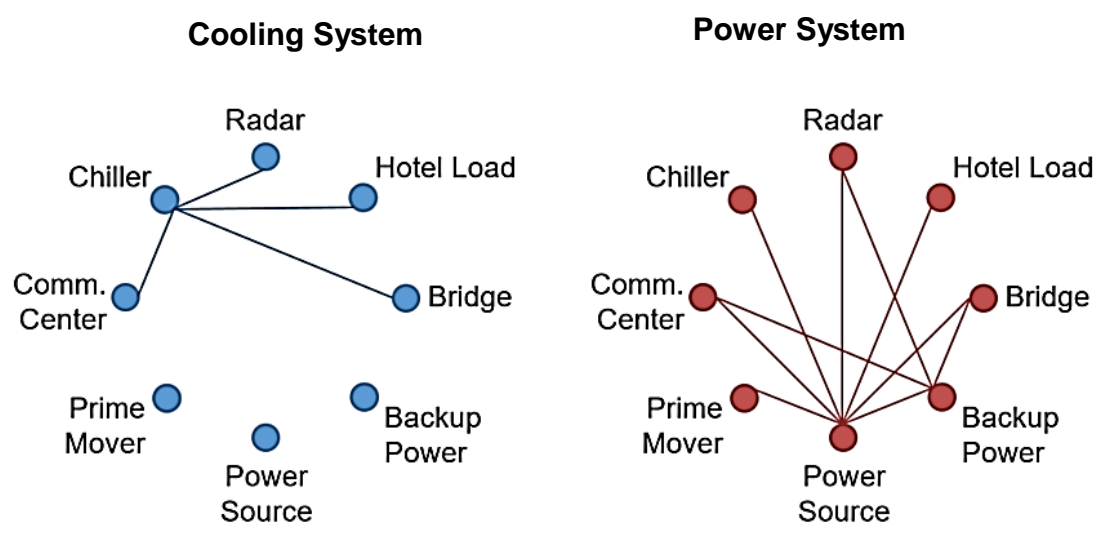
Figure 2: Logical architecture showing an implicit relationship between cooling and power

The operational architecture defines the temporal behavior of system. Temporal behavior is intended to capture what needs to happen through time to accomplish a given mission scenario. What systems are needed in which order, what processes and input/outputs are needed through time (like personnel movement or the charging of a capacitor). It characterizes the way systems and spaces are used over time for a given operational scenario, and the required system demands over time (their input and output needs). Since the operational architecture describes how a system is used over time, it also describes to some extent the human interaction with the system. More generally, it relates to the US Department of Defense definition of operational architecture: "description of the tasks, operational elements, and information flows required to accomplish or support a warfighting function" (Fry, 2001). For example, this may represent a load characterization based on operational use, or a survivability strategy that lays out what processes must take place to mitigate a damage scenario in the damaged or recoverability stages. An example of a visualization of operational architecture is presented in Figure 3. It consists of the operational need, the human activity and the machine system activity. The operational need describes to what extent the system is required over time. This can, for example, be the need for a weapon system to be deployed, or the need to use the steering gear in order to sail towards a desired course. The human activity results from the operational need. It includes the decisions made by the crew in order to fulfill the operational need. The machine system activity describes to what extent the actual hardware of the system is used over time. In this example the human activity and the machine system activity start at zero. However, they can also start above zero if 
the situation is continuously monitored. The operational need, human activity and machine system activity mutually influence each other and together form the operational architecture.

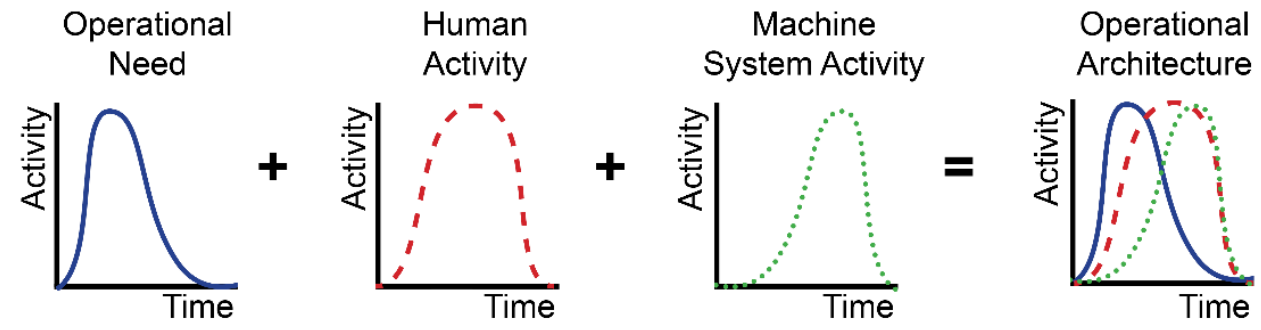

Figure 3: Example of a visualization for operational architecture, including the operational need, the human activity and the machine system activity of a distributed system.

\subsection{Interrelations between primary architectures}

The following discussion aims to show how the three primary architectures fit together to produce additional layers of information depth not captured by the primary architectures individually. There are four interrelations, defined as the physical solution, functional utilization, physical behavior, and system response. The authors have produced two representations of the architectural framework. Figure 4 presents a qualitative representation, showing how the three dimensions of the naval distributed systems problem can be decomposed and combined to understand different perspectives of the problem. Each overlap shows how information from the primary architectures can be combined to provide designers a deeper understanding of a potential design solution. Some of the authorship of the paper has questioned whether this model is actually sustainable. The basis of these concerns is that the physical, logical, and operational architectures each provide a different type of information, and thus from a set-theoretic perspective the intersection between them is empty. Therefore, an alternative 
graphical representation is presented at the end of this section (Figure 5). Meanwhile the original representation is developed further below as it is considered to provide some useful insights.

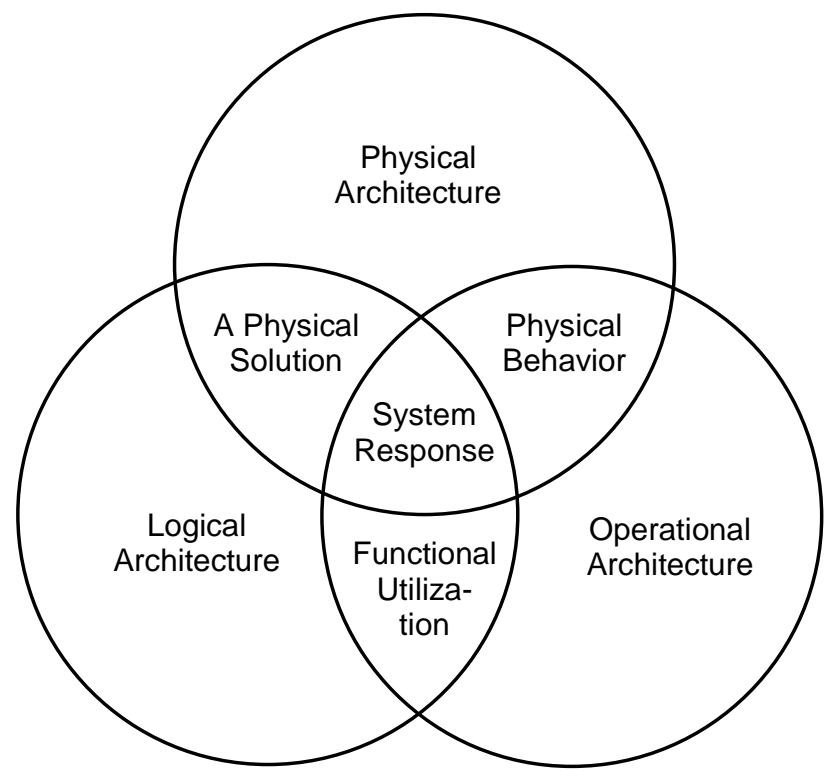

Figure 4: Visual representation of the architectural framework for naval distributed systems for a specific scenario.

The interrelation between the physical and logical architectures is defined as the physical solution, which describes what the system components look like in physical space of the ship, in the context of the chosen physical and logical architectures. This can include the physical layout out of the chilled-water plant, the radar, and the piping connecting the components together. The physical solution overlays the selected logical connections according to the spatial allocations (Shields et al., 2017).

The interrelation between the logical architecture and the operational architecture is the functional utilization. It characterizes the connection between system components and resource 
flows-required in time to fulfill a mission of interest. It specifies which systems are used or can be used over time from a given logical arrangement, to fulfill a specified mission. If Generator A breaks down, Generator B can be substituted to supply the required power to run a mission specific load. A system's functional utilization can be thought of as the system's load balance for a specified mission.

The physical behavior is the interrelation between the physical architecture and the operational architecture. It is a measure for defining the characteristics of a physical lay-out for a given mission of interest. It could capture interferences that occur as a result of undertaking a specified operational scenario. For example, electromagnetic interferences that occur if an operational scenario requires very high electrical loads, or the potential degradation of human performance when operating above a certain sea state. The physical performance also captures more grounded relations between physical attributes and performance attributes. For example, the physical size of a power generator is related to its power, so the maximum power requirements for a range of operational architectures will determine the appropriate physical architecture.

Combining the architectures and their interrelations creates a system response. This is a characterization of the performance or behavior of the system for a chosen physical layout, a chosen set of relations between components, and a chosen mission. By taking several instances of a primary architecture (ie. one catamaran and one mono-hull physical architecture) and analyzing each with respect to instances of the other two primary architectures, one gets a set of system responses, or the envelope of influence between architectures. The system response may be defined as the primary measure of performance or measure of effectiveness for the given 
system, in the specified operational context, and gives an overview of the distributed system. For example, after a hostile attack, the physical architecture will help determine which spaces suffered physical damage. The connectivity between systems, determined by the logical architecture will identify which systems will lose their connection, and thus their input. The operational architecture determines how systems are used and needed. Together, these architectures provides an overview of the system. The physical solution might change due to damage or isolation. The physical behavior identifies how a given physical configuration affects the system's capability after damage. The functional utilization might change to allow adequate operations with the system in a damaged state. Together, all three will determine the immediate damage, cascading failures, but also how systems can be reconfigured to mitigate damage propagation. This characterizes an overall response of the system in a selected design.

We now present the second representation of the architectural framework. This formal description of the framework takes into account the lack of set-theoretic interactions between primary architectures, and is illustrated in Figure 5. Despite that, the definitions of each architecture are identical to those of the first representation, presented in Figure 4. 


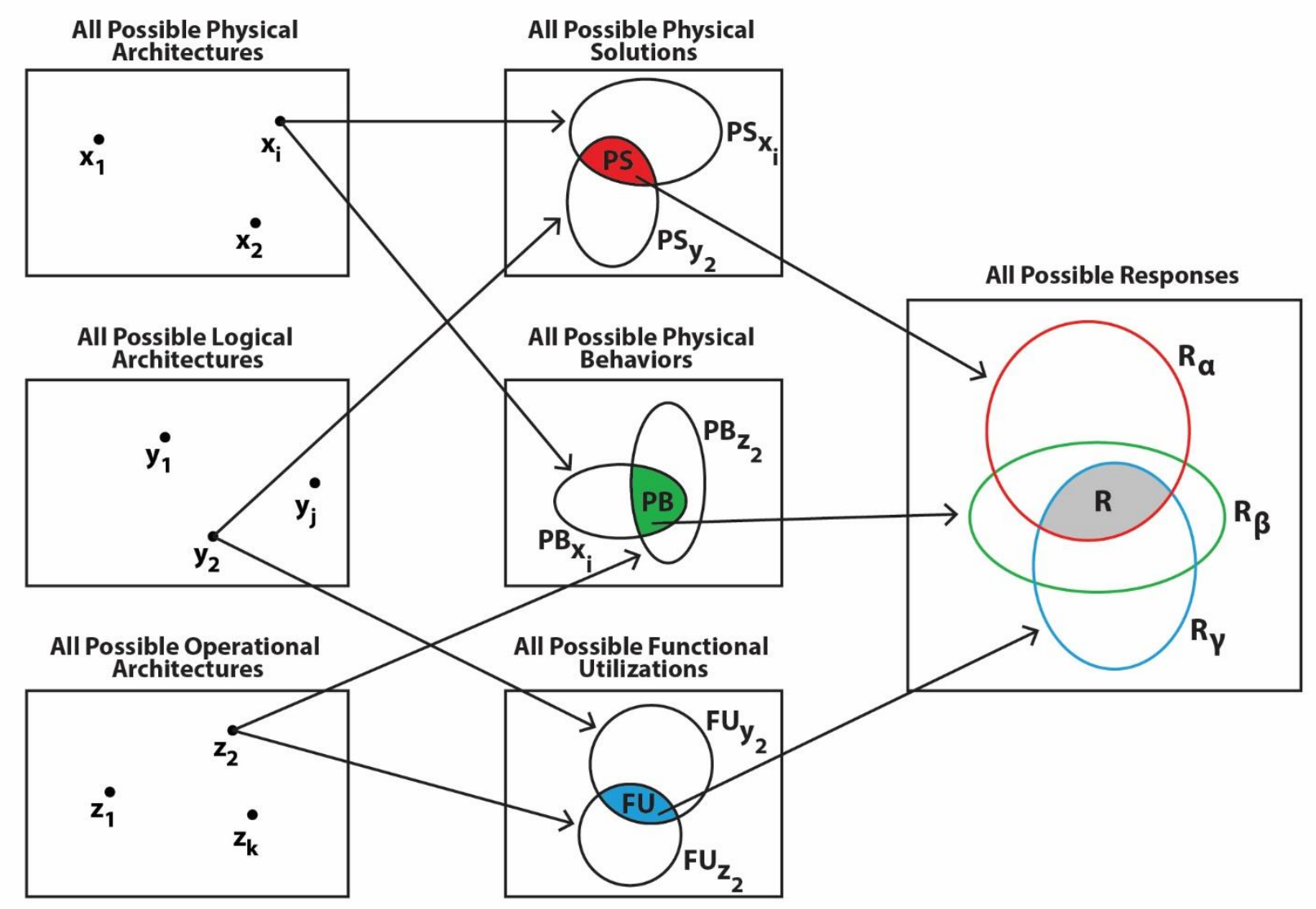

Figure 5: Formalized representation of the architectural framework for naval distributed systems. Rectangles in the left column represent the universe of all possible physical, logical, and operational architectures, respectively. The points in each rectangle represent instances of each architecture (i.e. $y_{1}$ is one possible logical architecture). Combining information from primary architectures provides information on the interactions between architectures. For example, systems in the logical architecture must be connected, but the physical architecture constrains how this routing can be implemented. The selected routing is one physical solution out of the set of possible physical solutions that can exist under the selected physical architecture and under the selected logical architecture. The filled red conic represents this set of possible physical solutions. In general, the conics represent subsets of their universe which exist under the 
element at the start of the arrow. Rectangles in the middle column represent the universe of all possible physical solutions, physical behaviors, and functional utilizations that can exist under any physical, logical or operational architecture. The right rectangle represents the universe of all possible responses. Designs associated with the filled red conic will exhibit a set of performance characteristics (the red circle $\mathrm{R}_{\alpha}$ ) related to the physical solution. Physical solutions existing under a different physical and logical architecture would have a different set of performance characteristics $\mathrm{R}_{\alpha}{ }^{\prime}$. The overall response $\mathrm{R}$ must account for the response, or performance characteristics, with respect to physical solution, physical behavior, and functional utilization. [END OF CAPTION]

The sets of physical architectures, logical architectures, and operational architectures are given by

$$
\begin{aligned}
& P A=\left\{x_{1}, x_{2}, x_{3}, \ldots, x_{i}\right\} \\
& L A=\left\{y_{1}, y_{2}, y_{3}, \ldots, y_{j}\right\} \\
& O A=\left\{z_{1}, z_{2}, z_{3}, \ldots, z_{k}\right\}
\end{aligned}
$$

Here, each $\mathrm{x}, \mathrm{y}, \mathrm{z}$ are different instances of a physical, logical, and operational architecture. For example, $\mathrm{x}_{1}$ and $\mathrm{x}_{2}$ could represent a mono-hull and double-hull configuration respectively. Let $\mathrm{PS}_{\mathrm{x}_{\mathrm{i}}}$ be the set of physical solutions possible under physical architecture $\mathrm{x}_{\mathrm{i}}$ and any logical architecture. That is, a given physical architecture can lead to a given set of physical solutions regardless of the logical architecture. Similarly, $\mathrm{PS}_{\mathrm{y}_{\mathrm{j}}}$ is the set of physical solutions possible under a specific logical architecture $y_{j}$ and a set of possible physical architectures. For example, the attack logical architecture shown in Figure 8 could be achieved in a mono-hull, as in Figure 
10 , or it could be achieved in a trimaran. These options are the physical solutions which belong to the set $\mathrm{PS}_{y_{\mathrm{j}}}$. Together, the selected logical architecture $\mathrm{y}_{\mathrm{j}}$ and the selected physical architecture $\mathrm{x}_{\mathrm{i}}$ provide the possible bounds on the set of possible physical solutions which are given by $\mathrm{PS}=\mathrm{PS}_{\mathrm{x}_{\mathrm{i}}} \cap \mathrm{PS}_{\mathrm{y}_{\mathrm{j}}}$. The physical solution is bounded by a 2-tuple of a physical architecture and a logical architecture and thus cannot be defined if one of them does not exist. Similarly, the set of physical behaviors possible under a given physical architecture $\mathrm{x}_{\mathrm{i}}$ and operational architecture $z_{k}$ is given by $P B=\mathrm{PB}_{x_{i}} \cap \mathrm{PB}_{z_{k}}$ where $\mathrm{PB}_{\mathrm{x}_{\mathrm{i}}}$ is the set of physical behaviors which exist under physical architecture $\mathrm{x}_{\mathrm{i}}$ and $\mathrm{PB}_{\mathrm{z}_{\mathrm{k}}}$ is the set of physical behaviors that exist under operational architecture $\mathrm{z}_{\mathrm{k}}$. The set of functional utilizations possible under a selected logical architecture $y_{j}$ and a scenario specific operational architecture $z_{k}$ is given by $\mathrm{FU}=\mathrm{FU}_{\mathrm{y}_{\mathrm{j}}} \cap \mathrm{FU}_{\mathrm{z}_{\mathrm{k}}}$

For the response $\mathrm{R}$, let $\mathrm{R}_{\alpha}$ be the set of overall system responses possible for the given set of physical solutions PS, $\mathrm{R}_{\beta}$ be the set of overall system responses for the given set of physical behaviors $\mathrm{PB}$, and $\mathrm{R}_{\gamma}$ be the set of overall system responses for the given set of functional utilizations FU. For example, the physical solution will dictate the robustness of a distribution system, influencing the set of responses $\mathrm{R}_{\alpha}$ possible under the chosen physical solution. The functional utilization, or load balance, will dictate the time dependent input and output the system needs and has. The set of responses $\mathrm{R}_{\gamma}$ will characterize the system with respect to its component connections, for a given time dependent operational scenario. The set of responses $\mathrm{R}_{\beta}$ for a given physical behavior, is related to the influence of the physical characteristics of a distributed system on a given operational scenario. All three sets of responses are required to 
evaluate the solution. That is, to get a complete characterization of the system's response R, responses possible under the given physical solution, physical behavior, and functional utilization must be considered. This is written as $R=R_{\alpha} \cap R_{\beta} \cap R_{\gamma}$.

\section{Requirements for Analysis Tools}

As with any framework, a set of design and analysis tools are necessary to properly analyze the various aspects of the system. While the objective of this paper is to outline the framework itself, it is nevertheless beneficial to include a brief consideration of the requirements for potential design tools. The requirements development for these design tools are outline in this section. While the development of these tools is considered an area of future work, this section also briefly discusses some potential tools being considered, with the caveat that many of them are still to be developed. No single design tool is capable of covering the entire framework. Instead, a set of tools is recommended to handle each part of the framework systematically. These tools must model hundreds or possibly thousands of diverse components. Figure 6 shows an example system, that shows the scope of this challenge. 


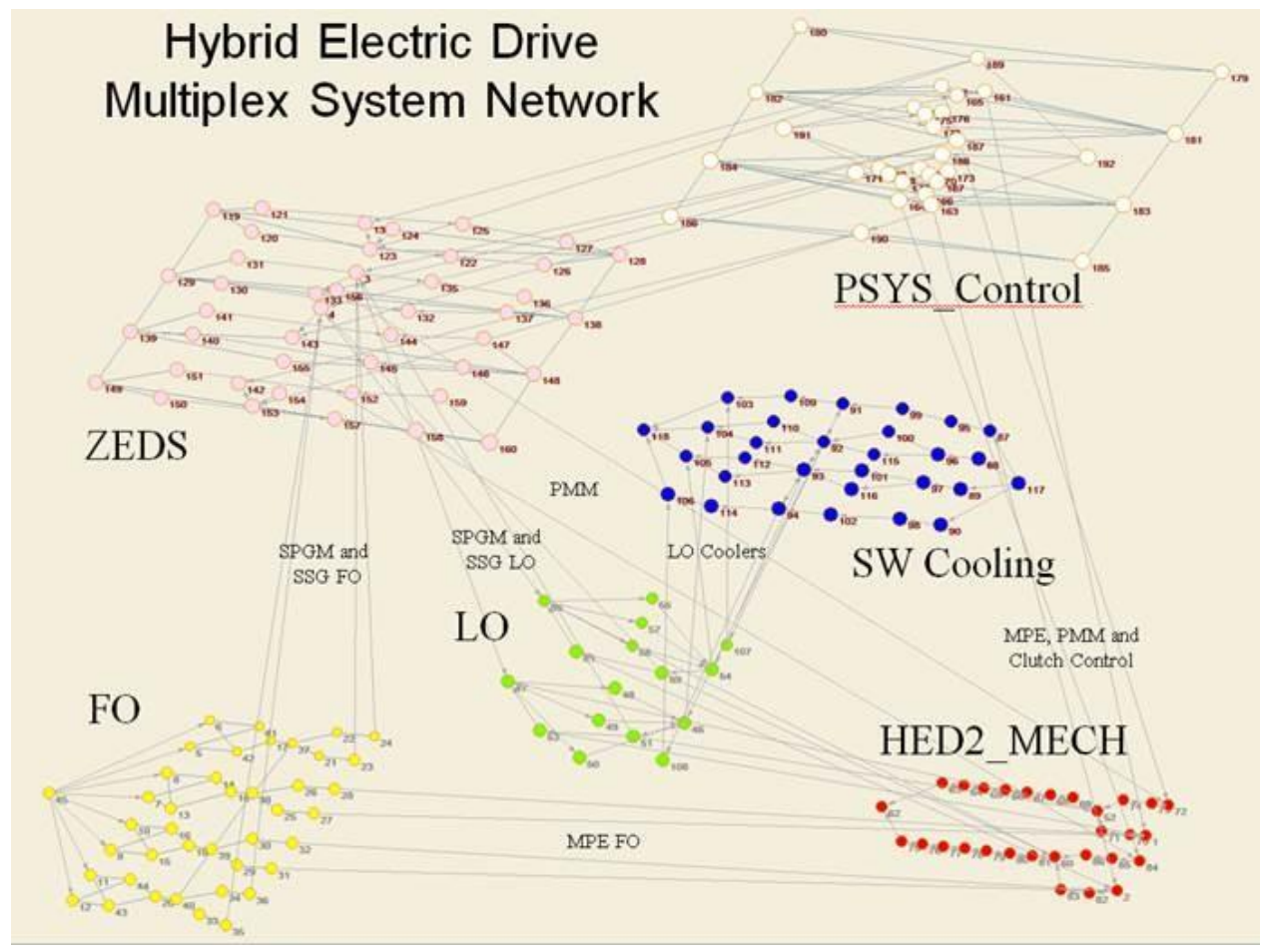

Figure 6: An example of a hybrid electric drive multiplex system network including mechanical, fuel oil, lube oil, cooling, zonal electrical distribution, and control components

To understand the physical architecture, design tools must be able to generate and analyze physical spaces. When in the concept phase, this should involve the ability to generate a vast number of various architectures to properly explore the design space (Andrews, 2013). Such tools that have been developed with this specific requirement in mind include the Design Building Block approach pioneered by Andrews (1998) and realized practically by Munoz and Forrest (2002) and Andrews and Pawling (2003), or the packing based approach developed by van Oers (2011), and Duchateau (2016). Additionally, network based tools are being developed to supplement these geometric layout based tools. Network tools require lower fidelity information, which allows ensemble analysis in the early stages of design with less information. 
Thus, it avoids predicating the design, since it require fewer assumption. Two current network tools are being investigated. The first to analyze the vulnerability of physical architectures with respect to internal blast, and the second to analyze the relationship between a selected physical architecture and the vessel's design complexity (Shields et al., 2016).

Understanding the logical architecture requires an understanding of the relationships and dependencies between the various elements of the system. This requirement lends itself to tools like network theory based metrics. Network theory has been used extensively to study the survivability of interdependent systems like power grids and communication networks (Buldyrev et al., 2010; Lü et al., 2016; Sterbenz et al., 2011; Newman, 2010). In ship design, Gillespie and Singer (2013); Rigterink et al., (2013); Collins et al. (2015); Parker and Singer, (2015); and Shields et al. (2016) have used networks extensively to study relationships and connections during various stages of the naval ship design process, including some studies focused specifically on distributed systems. Chalfant et al. (2017) are developing two logical architecture tools to study the operation and vulnerability of a total ship system: a deactivation block diagram algorithm and an architecture flow optimization expanded from Trapp (2015). Additional network based tools are being developed to study the combined influence of the physical and logical architectures on cascading failures.

Inherent in operational architecture are temporal variations and personnel requirements, which have to be met by requisite tool. Current research on studying the temporal aspects of operational architectures has looked to extend previous work on Markov processes as applied to ship design and operations (Niese, 2012; Kana, 2016). Markov processes provide a sound foundational 
structure that accounts for the stochastic uncertainty that may be present in temporal processes. Initial investigations into applying these tools to ship board survivability have looked into the use of both discrete and continuous time Markov processes to capture the temporal interplay between multiple ship systems during a damage scenario as well as the impact of individual component reliability on overall system performance and availability. From a personnel perspective, a distinction can be made between the logistical and non-logistical parts of operational processes. The logistical part requires connectivity between spaces and lends itself to a capacitated flow network-based approach, while the non-logistical part concerns a wider variety of information where a logical rules set approach can be pursued. The combination of the logistical and nonlogistical processes are used to evaluate the suitability of personnel requirements within the operational architecture. Developing tools to study the interactions of these architectures may involve combining various previously-existing tools, or the development of new ones.

\section{Framework Application Example}

When designing a new distributed ship system, clearly defining which aspects belong to each portion of the framework is important; however, it may be challenging. It is through this development and identification that insight will be gained about the system. In order to illustrate how the architectural framework can be applied to the analysis of a distributed system example, the use of a high energy weapon on a naval ship is presented. The related distributed system is limited to the weapon, the power source, the radar, the chiller, the combat information center, the cables and pipes. For this example, the physical architecture consists of the constraining ship architecture and the locations of the appropriate components, as shown in Figure 7. 


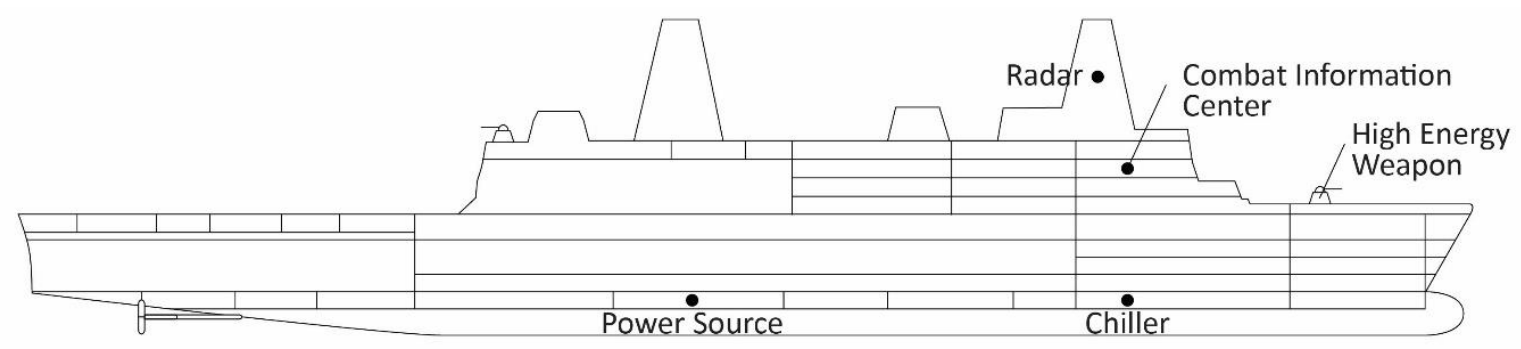

Figure 7: An example physical architecture showing the constraining architecture and the location of appropriate components.

A logical architecture describes the relationship between the system components. In this case it is the single-line diagram of a set of required power, information, and cooling systems associated with the weapon system. It is a schematic overview of the required connections between generators, weapon, radar, chiller, and cables and the associated electrical elements, as shown in Figure 8. System components and their connections are organized by services that must exist for the attack system to function: powering, information, and cooling. 


\section{Attack System Logical Architecture}

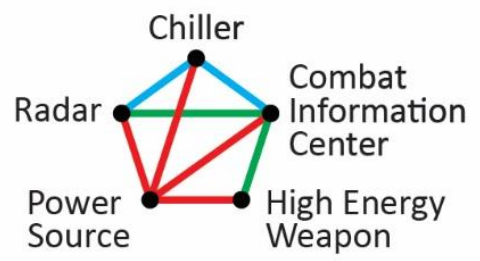

Information Logical Architecture

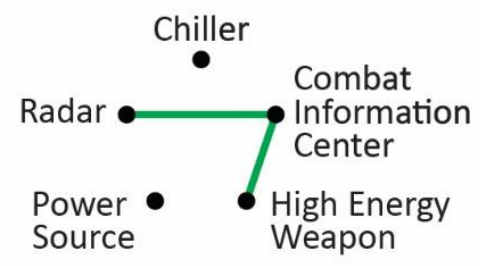

Powering Logical Architecture

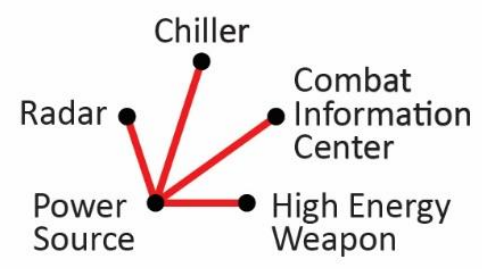

\section{Cooling Logical Architecture}

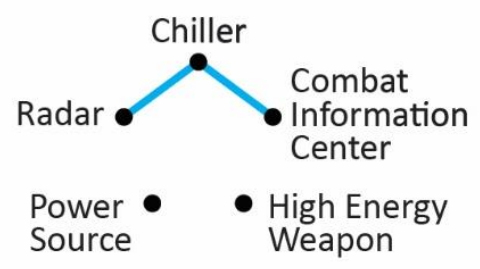

Figure 8: Logical architectures showing the connections between components of an example of a distributed system. Connections will differ based on the required services of the system.

The operational architecture is a way of representing how the system is employed over time. It can be visualized using a graph of the operational need and the system activity (both human systems and machine systems) as a function of time for a specific mission scenario. This graph can be dependent on the system of interest and external factors appropriate to a selected operational scenario. For this case, the associated graph is presented in Figure 9. The weapon operating process consists of several phases. In Phase 1 an operational need for employing the weapon arises. This can be a hostile attack. The activity of the machine system, which is the weapon in this case, is still zero. The human activity is slightly above zero, since the humans are always monitoring the situation prior to taking decisions. In Phase 2 the crew has observed the hostile attack. The human activity increases because the crew has to decide how to react to the 
attack. The crew decides to employ the weapon in Phase 3. The machine system activity therefore increases. The human activity decreases slightly because the machine system has partly taken over the action. In Phase 4 the hostile attack is effectively combatted. The human activity increases again because the crew has to decide how next to act. It is decided that the external threat is over (Phase 5). The operational need decreases to zero and the weapon is deactivated. The human activity is reduced to the original level.

It is characteristic of an operational architecture that there will be a certain degree of time lag between operational need, human activity and system activity. Furthermore, the plot lines clearly illustrate the balance between the operational need and the system activity, and how it develops over time for that scenario.

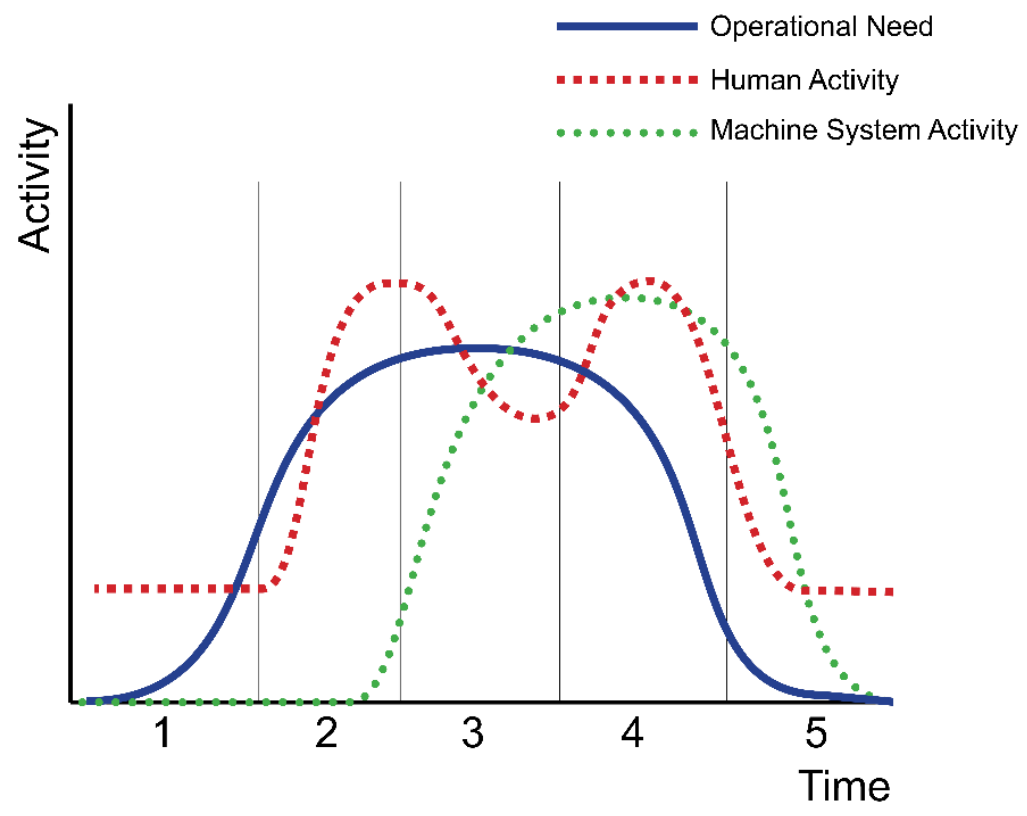

Figure 9: Example of operational architecture with operational need, human activity and machine system activity as functions of time. The numbers 1-5 denote the phases of activity over time. 
Subsequently, the interrelations of the diagram can be determined. The physical solution, which links the location of the attack system components to the required connections that must exist between them, includes the chosen components, and the cable and pipe routing between them. It is likely to be restricted by the design constraints of the physical architecture, such as the placement of bulkheads. The physical solution of the attack system is shown in Figure 10. The attack system is dependent on powering, information, and cooling services to function. A breakdown of any of these services, through a failure of the system components, of their human or system management, or of their routing could cause the system to fail or exhibit a degraded performance. 


\section{Attack System Physical Solution}

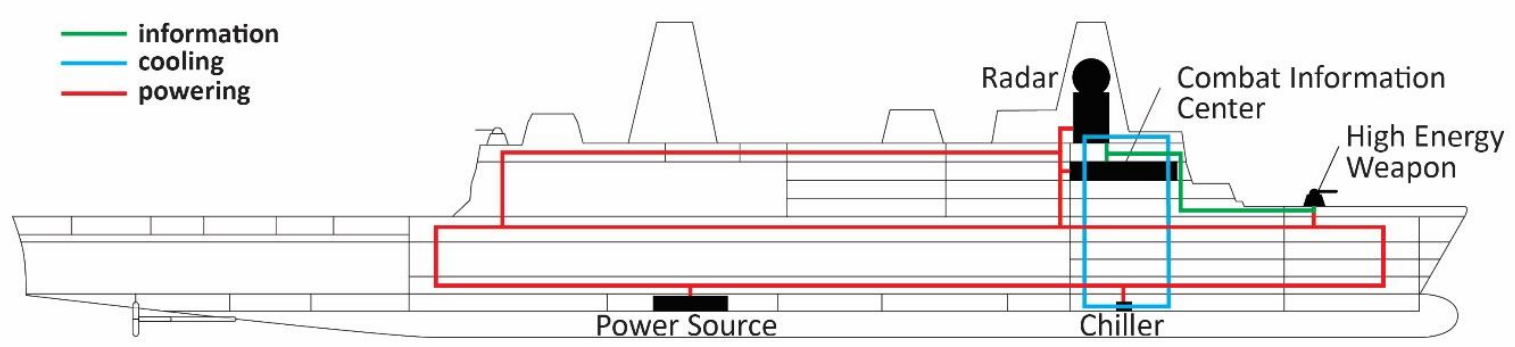

Powering Physical Solution

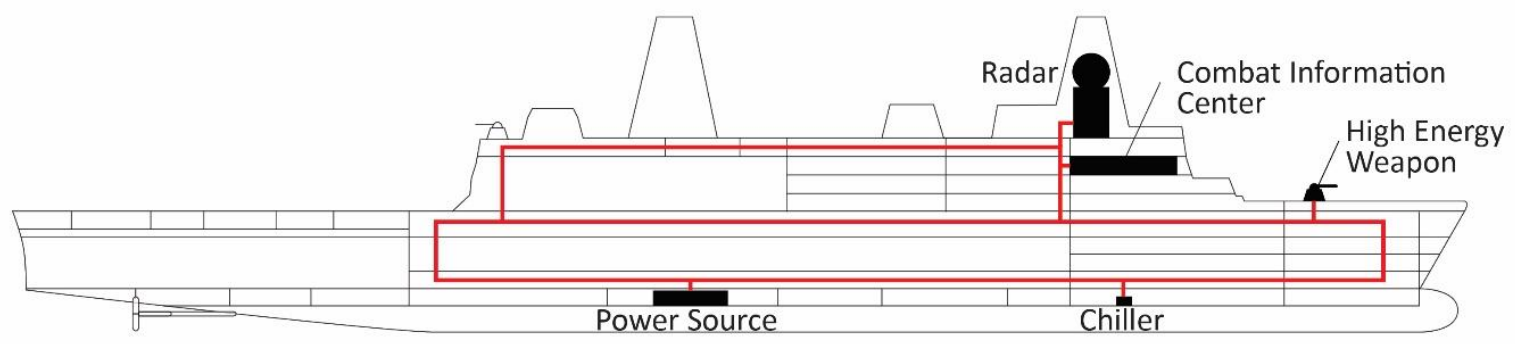

Information Physical Solution

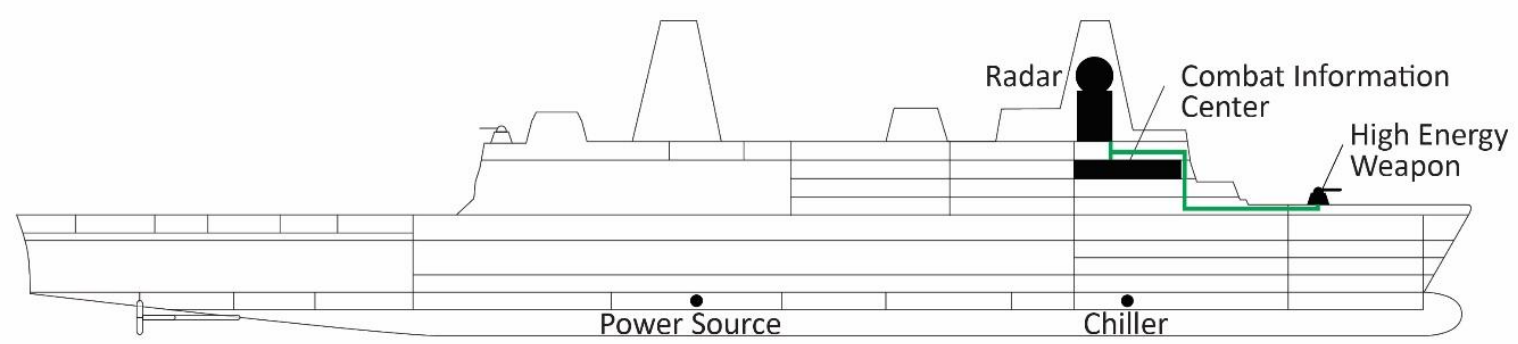

\section{Cooling Physical Solution}

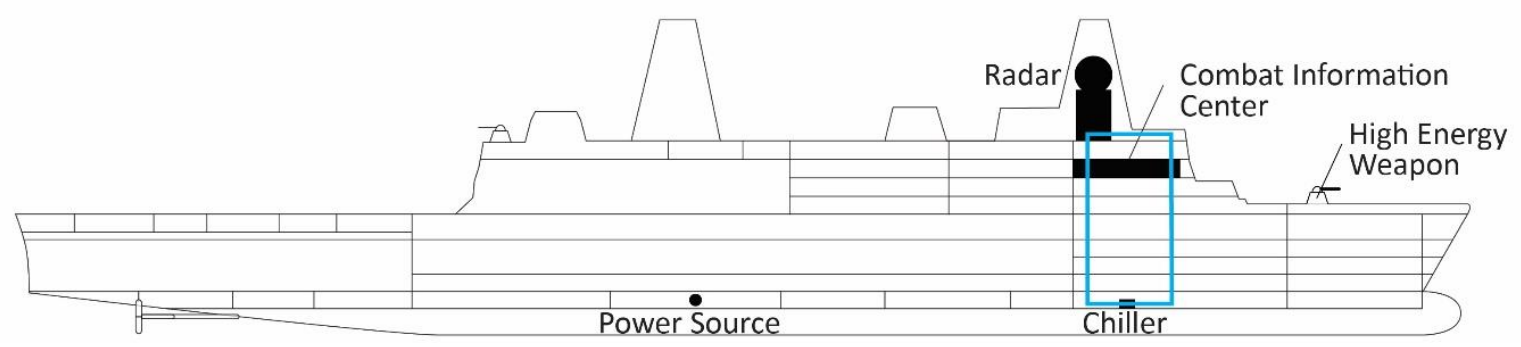

Figure 10: An example physical solution showing a possible set of connections and spatial routings between components of a distributed system. Component connections are organized by service. 
The overlap between the single-line diagram (logical architecture) and the specific power demand (operational architecture) is the functional utilization of the system needed to power a high energy weapon in a specific scenario. It is the load balance for this electrically based system. The physical behavior links the location and size of the weapon and generator to the power demand, which influences both the generator size, and the physical and electromagnetic footprint of the cables in this case. Due to high power requirements necessary for high energy weapons, the physical size of the power source may limit its location on the vessel, thus highlighting the relationship to the physical architecture and the potential need to evaluate different options for the ship's overall architecture and that of the relevant distributed systems. Additionally, in the above example, the operational architecture dictates large hangars for vehicle stowage, movement and transport, which limits the ability to route a large number of systems straight up from the engine room through the hangars. The operational architecture thus influences the physical architecture through the constraining topology and size demands will influence the physical architecture and vice versa. The response of the system is the power distribution. This describes both the magnitude of the power of the system and how it is distributed through the system to meet the envelope of the scenarios and logics. The graphical representation of the framework for this example is presented in Figure 11. 


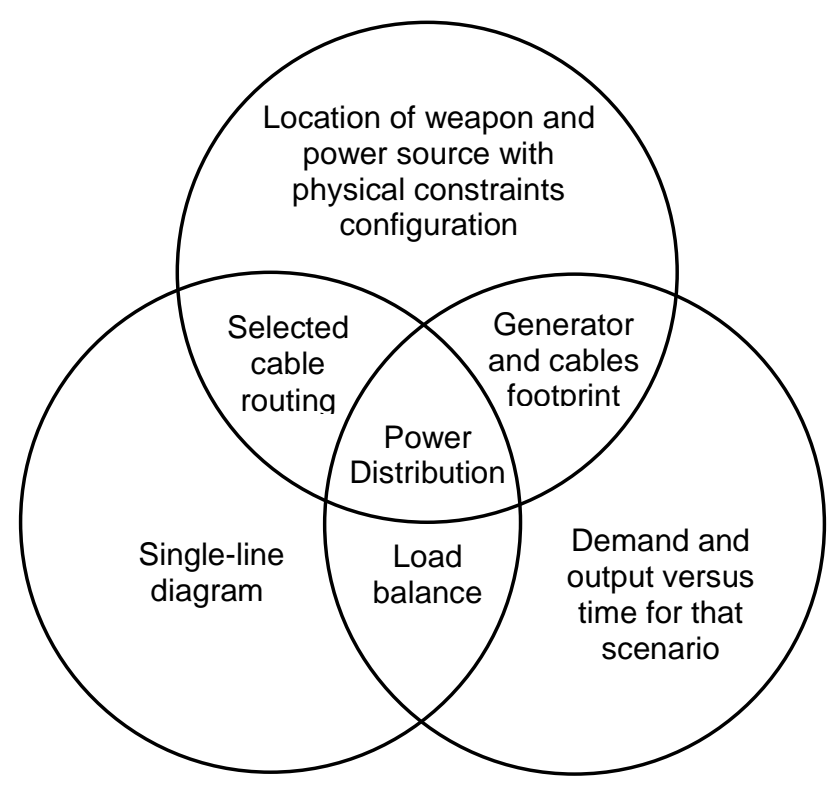

Figure 11: A specific example architectural framework for a simple system used to power a high energy weapon.

The framework can be used to evaluate changes in the distributed system architecture due to shifting requirements. For instance, if there is a need to fire the weapon, the operational architecture is influenced, since the power demand increases. This could influence the load balance, the generator size and the power distribution; however, the effects will be very dependent on the configuration of the power distribution system which must be considered from the physical, logical, and operational points of view. It can also be deduced that the generator size is related to the physical architecture. Though increasing the generator size can fulfill the power demand, it may not be a solution that is suitable for practical applications. It might make more sense to focus on the load balance. This should be able to be shown from inspecting the single-line diagram of the system. The increase in power demand could be obtained by adjusting the logical architecture, such as activating switches. By adopting a holistic view of distributed 
systems, one can start to analyze the influences of the primary architectures' configurations on one another.

\section{Conclusions}

This paper has introduced an architectural framework for designing and analyzing distributed systems, within the context of naval ship survivability. The increasing number and importance of distributed systems aboard naval vessels has led to major increases in vessel complexity. The design and analysis of architectures of distributed systems will become an important design driver in modern naval ships; thus, new tools and methods are required at the concept design phase of vessels whose architecture is likely to be dominated by future distributed systems. The framework presented in this paper aims to fill this gap by providing the basis to better investigate the coupling between the various architectural properties of a vessel and its distributed systems, and their relationship to the vessel's performance. The physical, logical, and operational characteristics of a vessel, with regard to distributed ship systems, have been addressed, as well as their interactions, providing designers a more holistic view of the ship, its distributed systems, and their behaviours under specific scenarios. Having this knowledge in early-stage design will give designers a better understanding of the changing design drivers of future naval vessels which will have a higher reliance on interconnected distributed systems. It will also provide designers with a better basis for evolving appropriate tools needed to take advantage of the new opportunities. These opportunities will be realised by these distributed systems and by recent technology developments, such as all-electric power and propulsion systems. 


\section{Future Work}

The research presented here is part of a multi-year multi-university project, and thus several items have been identified for future work. The framework itself will serve as a foundation to our work, and the authors will work to develop and refine various aspects of it moving forward. Specifically, this future development may be split between application and theory. For application, the authors are working to develop case studies explaining the framework in practice. On top of the development of a detailed case study, there is also the need to document a list of tool requirements for analyses of each portion of the framework, including information exchange, and to outline recommended standards for practice. On the theoretical side, the authors are working to extend this framework to include relations between systems themselves, as well as systems-of-systems and sub-systems. Later, applications and case studies will be developed focusing specifically on the multi-layered distributed ship systems architectural framework.

\section{Project Biography}

This research stems out of a multinational university collaboration involving: University of Michigan, Delft University of Technology, University College London, and Virginia Tech. These partners gratefully acknowledge the funding of this Naval International Cooperative Opportunities in Science and Technology Program (NICOP), sponsored by Ms. Kelly Cooper of the US Navy Office of Naval Research, which is a follow-on of a previously successful collaboration. The original work was focused on studying methods for generating and analyzing general arrangements in early stage ship design. This follow on project extends that work to study the relationship between distributed system design and general arrangements, in the context of ship survivability. The initial task of this recent project was to develop a common 
vernacular between the universities and a framework to which all parties could agree upon. That vernacular and framework are the foundation of this article.

\section{Acknowledgements}

The authors would like to thank Ms. Kelly Cooper and the Office of Naval Research for their support on this collaborative study of the relationship between general arrangements and distributed systems with a focus on survivability, funded under the Naval International Cooperative Opportunities in Science and Technology Program (NICOP) contract number N00014-15-1-2752.

\section{References}

1. Altosole, M., Dubbioso, G., Figari, M., Viviani, M., Michetti, S., Millerani Trapani, A., 2010. Simulation of the Dynamic Behaviour of a CODLAG Propulsion Plant. Warship 2010: Advanced Technologies in Naval Design and Construction. London, UK

2. Andrews, D., 1998. A Comprehensive Methodology for the Design of Ships (and Other Complex Systems). Proceedings of the Royal Society: A, 454: 187-211.

3. Andrews, D., 2011. Marine Requirements Elucidation and the Nature of Preliminary Ship Design. Transactions of the Royal Institution of Naval Architects Part A:

International Journal of Maritime Engineering, 153 (Part A1). 
4. Andrews, D., 2012. Art and Science in the Design of Physically Large and Complex Systems. Proceedings of the Royal Society A: Mathematical, Physical and Engineering Sciences, 468: 891-912.

5. Andrews, D., 2013. The True Nature of Ship Concept Design - And what it means for the future development of CASD. In Proceedings of the Conference of Computer Applications and Information Technology in the Maritime Industries (COMPIT). Pages 33-50, Cortona, Italy.

6. Andrews, D. and Pawling, R., 2003. SURFCON - A 21st Century ship design tool. In International Marine Design Conference (IMDC). Athens, Greece

7. Britton, B., 2016. Britain's Royal Navy warships are breaking down because sea is too hot, CNN World.

8. Brown, A., Waltham-Sajdak J., 2015. Still Reengineering the Naval Ship Concept Design Process, Naval Engineers Journal, 127 (1):49-61.

9. Buldyrev, S.V., Parshani, R., Paul, G., Stanley, H.E, Havlin, S., 2010, Catastrophic Cascade of Failures in Interdependent Networks, Nature, 464(7291):1025-8

10. Chalfant, J., 2015. Early-Stage Design for Electric Ship, Proceedings of the IEEE 103 (12):2252-2266. 
11. Chalfant, J., Chryssostomidis, C., Angle, M., 2010. Study of Parallel AC and DC Electrical Distribution in the All-Electric Ship, In Grand Challenges in Modeling and Simulation (GCMS10), Ottawa, Canada

12. Chalfant, J. Chryssostomidis, C., Synder, D., Parsons, M. A., and Brown, A., 2017. Graph Theory Applications in Focus-compliant Ship Design. In 2017 IEEE Electric Ship Technologies Symposium, Crystal City, VA, USA.

13. Chandrasegaran, S., Ramani, K., Sriram, R., Horváth, I., Bernard, A., Harik, R., Gao, W., 2013. The Evolution, Challenges, and Future of Knowledge Representation in Product Design Systems. CAD Computer Aided Design, 45(2): 204-228.

14. Collins, L., Pawling, R., and Andrews, D., 2015. A New Approach for the Incorporation of Radical Technologies: Rim Drive for Large Submarines, In International Marine Design Conference (IMDC), Tokyo, Japan.

15. Cramer, A., Sudhoff, S., Zivi, E., 2009. Evolutionary Algorithms for Minimax Problems in Robust Design. IEEE Transactions on Evolutionary Computation, 13(2): 444-453.

16. Desfray, P., 2008. Enterprise Architecture: Practical Guide to Logical Architecture. Report, Softeam Consulting Team.

17. Dobson, A., 2014. Cost Prediction Via Quantitative Analysis of Complexity in U.S. Navy Shipbuilding, Ph.D. Thesis, Massachusetts Institute of Technology. 
18. Doerry, N., 2006. Zonal Ship Design, Naval Engineers Journal, 118 (1):39-53.

19. Doerry, N., 2007. Designing Electrical Power Systems for Survivability and Quality of Service. Naval Engineers Journal, 119 (2): 25-34.

20. Doerry, N., 2014. The Evolution of the Electric Warship. Naval Engineers Journal, 126 (1):173-186.

21. Doerry, N. and Fireman H., 2006. Designing all Electric Ships. In Proceedings of the Ninth International Marine Design Conference (IMDC), pages 475-497, Ann Arbor, MI.

22. Duchateau, E., 2016. Interactive Evolutionary Concept Exploration in Preliminary Ship Design. PhD thesis, Delft University of Technology, the Netherlands.

23. Ehlers, S., Asbjornslett, B., Rodseth, O. J., Berg, T., 2014. Maritime-Port Technology and Development, CRC Press, Boca Raton, FL.

24. Fiedel, E., Chalfant, J., Chryssostomidis, C., 2011. Cooling System Early-Stage Design Tool for Naval Applications. In Proceedings of the 2011 Grand Challenges on Modeling and Simulation Conference, Society for Modeling \& Simulation International.

25. Ferreiro, L.D, Stonehouse, M.H., 1991. A Comparative Study of US and UK Frigate Design. SNAME Transactions, 99: 147-175. 
26. Fry, S.A., 2001. Dictionary of Military and Associated Terms. United States Department of Defense, as amended through April 2010.

27. Gillespie, J. and Singer, D., 2013. Identifying Drivers of General Arrangements Through the Use of Network Measures of Centrality and Hierarchy, Ocean Engineering, 57: 230239.

28. Gortney, W.E., 2010. Dictionary of Military and Associated Terms. United States Department of Defense, as amended through June 2015.

29. Greig, A.R., Coombes, J., Andrews, D., Pawling, R., 2009. Modelling the Heat Distribution in a Warship. In World Maritime Technology Conference (WMTC), Mumbai India

30. Kana, A.A., 2016. Enabling Decision Insight by Applying Monte Carlo Simulations and Eigenvalue Spectral Analysis to the Ship-Centric Markov Decision Process Framework. $\mathrm{PhD}$ thesis, University of Michigan, USA.

31. Kassel, B., Cooper, S., Mackenna, A., 2010. Rebuilding the NAVSEA Early Stage Ship Design Environment. In ASNE 2010.

32. Lü, L., Chen, D., Ren, X., Zhang, Q., Zhang, Y., Zhou, T., 2016. Vital Nodes Identification in Complex Networks, Physics Reports, 650:1-63 
33. Miroyannis, A., 2006. Estimation of Ship Construction Costs, PhD Thesis, Massachusetts Institute of Technology, USA.

34. Munoz, J. and Forrest, C., 2002. Advantages of Software Integration from Initial Design Through to Production Design. In International Conferences on Computer Applications in Shipbuilding (ICCAS), Malmo, Sweden

35. Newman, M.E.J., 2010. Networks: An Introduction, Oxford University Press, Oxford, UK.

36. Niese, N.D., 2012. Life Cycle Evaluation under Uncertain Environmental Policies Using a Ship-Centric Markov Decision Process Framework. PhD thesis, University of Michigan, USA.

37. Ouroua, A., Jackson, J.R., Beno, J.H., Thompson, R.C, Schroeder, E., 2007. Modeling and Simulation of Electric Ships' Power System Components and their Interactions. In Summer Computer Simulation Conference, San Diego, USA

38. Parker, M. and Singer, D., 2015. Analyzing the dynamic behavior of marine design tools using network theory. Ocean Engineering, 106: 227-237.

39. Pawling, R., Andrews, D., Piks, R., Singer, D., Duchateau, E., and Hopman, H., 2013. An Integrated Approach to Style Definition in Early Stage Design. In Conference on 
Computer Applications and Information Technology in the Maritime Industries

(COMPIT), pages 248-263, Cortona, Italy.

40. Pawling, R., Morandi, R., Andrews, D., Shields, C., Singer, D., Duchateau, E., Hopman, H., 2014. Manifestation of Style and it Use in the Design Process. In Conference on Computer Applications and Information Technology in the Maritime Industries (COMPIT), pages 209-223, Redworth, UK.

41. Reason, J., 1990. Human Error, first ed. Cambridge University Press, Cambridge, England

42. Rigterink, D., 2014. Methods for Analyzing Early Stage Naval Distributed Systems Designs, Employing Simplex, Multislice, and Multiplex Networks. PhD Thesis, University of Michigan, USA.

43. Rigterink, D., Piks, R., and Singer, D., 2013. The Use of Network Theory to Model Disparate Ship Design Information. In Practical Design of Ships and Other Floating Structures (PRADS), pages 109-117, Changwon, Korea.

44. Slabodkin, G., 1998. Software glitches leave Navy smart ship dead in the water. Government Computer News.

45. Shields, C., Sypniewski, M., and Singer, D., 2016. Understanding the Relationship Between Naval Product Complexity and On-board System Survivability Using Network 
Routing and Design Ensemble Analysis. In Practical Design of Ships and Other Floating Structures (PRADS), pages 219-225, Copenhagen, Denmark.

46. Shields, C., Rigterink, D., and Singer, D., 2017. Investigating Physical Solutions in the Architectural Design of Distributed Ship Service Systems. Ocean Engineering 135: 236245.

47. Sterbenz, J.P.G., Çetinkaya, E.K., Hameed, M.A., Jabbar, A., Qian, S., Rohrer, J.P., 2013. Evaluation of Network Resilience, Survivability, and Disruption Tolerance: Analysis, Topology Generation, Simulation, and Experimentation. Telecommunications Systems, 52:705-736.

48. TOGAF, 2011. The Open Group Architecture Framework (TOGAF) Version 9.1, an Open Group Standard. http://www.opengroup.org/ (accessed 01.10.16).

49. Trapp, T., 2015. Shipboard Integrated Engineering Plant Survivable Network Optimization, Ph.D. Thesis, Massachusetts Institute of Technology, USA.

50. van Oers, B., 2011. A Packing Approach for the Early Stage Design of Service Vessels. PhD Thesis, Delft University of Technology, the Netherlands.

51. Whitelegg, I., Bucknall, R.W.G., Thorp, B.T., 2015. On Electric Warship Power System Performance When Meeting the Energy Requirements of Electromagnetic Railguns, Journal of Marine Engineering \& Technology, 14:2, 85-102, 


\section{Appendix: Nomenclature}

This project identified the need to define several terms related to distributed systems, general arrangements, and survivability. This was done because many of these terms have similar meanings in other fields, and there was a desire to maintain consistency and clarity throughout the duration of this project. The authors have tried to retain the definitions found in relevant literature; however, some modifications were necessary. In some cases, entirely new definitions were developed. Definitions from literature have been cited accordingly.

Architectural framework: A foundational structure, or set of structures, which can be used for developing a broad range of different architectures. It should describe a method for designing a target state of the enterprise in terms of a set of building blocks, and for showing how the building blocks fit together. It should contain a set of tools and provide a common vocabulary. It should also include a list of recommended standards and compliant products that can be used to implement the building blocks (TOGAF, 2011).

Component: A part or combination of parts having a specific function, which can be installed or replaced only as an entity. [Definition 2, as used in logistics] (Gortney, 2010).

Distributed system: A specific type of system that is disbursed throughout the vessel.

Functional utilization: The characterization of the connection between system components, in the context of fulfilling the mission of interest, e.g. the load balance between the different system components.

Logical architecture: A description of the connections between system components, from a macroscopic view, by focusing on interactions and flows exchange, and by structuring it into larger-scale modules (adapted From Desfray, 2008).

Operational architecture: A description of the tasks, operational elements, and information flows required to accomplish or support a warfighting function in time (Fry, 2001). 
Physical architecture: Spatial architecture describing the ship arrangements, and the physical attributes of components and their position in space.

Physical behavior: A measure for defining the characteristics of a physical lay-out for a given mission of interest as defined in the operational architecture.

Physical solution: The system design solutions which are present in physical space with allocated components and connections.

Recoverability: The prospects of recovery given damage.

Response: The combined response of system due to interactions between the physical architecture, the logical architecture, and the operational architecture. This may include the primary measures of effectiveness (MOEs) or measures of performance (MOPs).

Survivability: All aspects of protecting personnel, weapons, and supplies while simultaneously deceiving the enemy (Gortney, 210). Note: we propose this alternate definition, "the impact of impulses on ship processes and architectures, and what responses can be taken to mitigate losses in personnel and performance."

Susceptibility: The likelihood of a hit.

System: A functionally, physically, and/or behaviorally related group of regularly interacting or interdependent elements; that group of elements forming a unified whole (Gortney, 2010).

Vulnerability: The characteristics of a system that cause it to suffer a definite degradation (incapability to perform the designated mission) as a result of having been subjected to a certain level of effects in an unnatural (man-made) hostile environment. [Definition 2] (Gortney, 2010). 